\title{
The Travelling Wave Solutions of KdV-Burger Equations
}

\author{
Pinchao Meng ${ }^{a}$, Weishi Yin ${ }^{b}$ \\ School of Science, Changchun university of Science and Technology, Jilin 130022, P.R.China \\ amengpc@cust.edu.cn,b16153586@qq.com
}

\begin{abstract}
This paper is concerned with the existence of exact traveling wave solutions of some nonlinear evolution equations by the tanh-function method. The validity and reliability of this method is demonstrated by applying it to the KdV-Burger equations.
\end{abstract}

Keywords: Traveling wave solutions; Tanh-function method; KdV-Burger equations.

\section{Introduction}

Nonlinear phenomena play a fundamental role in applied mathematics, physics, power electronics, oscillatory systems, earthquakes and tremor recently. The study of nonlinear partial differential equations in modelling physical phenomena has become an important tool. The investigation of the travelling wave solutions plays an important role in nonlinear sciences. There are usually no analytical solutions for these problems, especially when the nonlinear terms are complicated. Therefore, it is practical importance to find travelling solutions.

The methods of looking for exact traveling wave solutions of nonlinear evolution equations, has been tremendous development in recent decades, such as Hirota's bilinear technique [5], MSE method [9],F-expansion method [13,19], the Painlve expansion method [18]. In 1990s, Huibin and Kelin [7] proposed a new method. The main idea of this method is taking hyperbolic tangent function of the power series as possi-ble traveling wave solutions of the nonlinear evolution equations. Then they substituted the power series directly to $\mathrm{KdV}$ equation, and obtained the coefficients of the power series. However this method involved very complicated algebra computation. In order to reduce the complex algebra computation, Malfiety [10-12] proposed the tanh-function method. Fan et al [3] proposed the extended hyperbolic tangent method, which replace the tanh-function by the solutions of Riccati equation. In [1, 4, 14-16], using the tanh function method, they got the exact form of traveling wave solutions of various types of evolution equations.

\section{The Tanh-function Method}

Let's consider the nonlinear partial differential equations

$N\left(u, u_{t}, u_{x}, u_{x x}, u_{x x x} \cdots\right)=0$,

Where $u(x, t)$ is the real function on $R^{2}$. At first, we assume the traveling wave solutions of (2.1) are the form of

$$
u(x, t)=U(\omega)=U(c(x-v t)) \text {, }
$$

With the velocity $v$, and the constant $c$. Submitted (2.2) into (2.1), we can get the ODEs about $\omega$

$$
N\left(U, U^{\prime}, U^{\prime \prime}, U^{\prime \prime \prime}, \cdots\right)=0 \text {. }
$$

Second, we assume the possibly traveling wave solutions can be written

$$
u(x, t)=U(\omega)=H(Y)=\sum_{i=0}^{K} a_{i} Y^{i},
$$

Where $Y=\tanh (\omega)=\frac{e^{\omega}-e^{-\omega}}{e^{\omega}+e^{-\omega}}$, the highest order $K$ will be determined late.

Then we can get

$$
\frac{d Y}{d \omega}=1-Y^{2}, \frac{d U}{d \omega}=\left(1-Y^{2}\right) H^{\prime}, \frac{d^{2} U}{d \omega^{2}}=\left(1-Y^{2}\right)\left(-2 Y \cdot H^{\prime}+\left(1-Y^{2}\right) H^{\prime \prime}\right) .
$$


$\frac{d^{3} U}{d \omega^{3}}=\left(1-Y^{2}\right)\left(6 Y^{2}-2\right) H^{\prime}-6 Y\left(1-Y^{2}\right) H^{\prime \prime}+\left(1-Y^{2}\right)^{2} H^{\prime \prime \prime}$,

Submitted above equations into (2.3), we can get the ODEs with $Y$

$N\left(Y, H, H^{\prime}, H^{\prime \prime}, H^{\prime \prime \prime}, \cdots\right)=0$,

Where $H^{\prime}=\frac{d H}{d Y}$. To determine the parameter $K$, we usually balance the nonlinear term and the highest order derivative term in equation (2.5). Then, we submitted (2.4) (with the determined $K$ ) into (2.5), and get the polynomial equation with $Y$, Collecting all the coefficients of power of $Y$; and letting the coefficients of each power of $Y$ to be vanished,we can determined all the coefficient $a_{1}, a_{2}, \cdots, a_{k}$. According to (2.4), we can get the traveling wave solutions of (2.1).

\section{Application}

We consider the KdV-Burger equations

$u_{t}+\alpha u u_{x}+\beta u_{x x x}+\gamma u_{x x}=0$,

Where $\alpha, \beta$ are non-zero constants. Submitting $u(x, t)=U(\omega)=U(c(x-v t))$ into (3.1), we get

$-c v U^{\prime}+\alpha c U \cdot U^{\prime}+\beta c^{3} U^{\prime \prime \prime}+\gamma c^{2} U^{\prime \prime}=0$.

Integrating the above equation, we have

$-v U+\frac{1}{2} \alpha U^{2}+\beta c^{2} U^{\prime \prime}+\gamma c U^{\prime}=0$.

In view of (2.4), proceeding as before, we obtain

$-v H+\frac{1}{2} \alpha H^{2}+\beta c^{2}\left(1-Y^{2}\right)\left[-2 Y H^{\prime}+\left(1-Y^{2}\right) H^{\prime \prime}\right]+\gamma c\left(1-Y^{2}\right) H^{\prime}=0$.

Balancing the terms $Y^{4} H^{\prime \prime}$ with $H^{2}$, we get $K=2$ So, we can obtain

$$
H(Y)=a_{0}+a_{1} Y+a_{2} Y^{2}, H^{\prime}=a_{1}+2 a_{2} Y, H^{\prime \prime}=2 a_{2} \text {. }
$$

Submitting it into (3.2), we obtain the following algebraic equations:

$$
\left\{\begin{array}{c}
-v a_{0}+\frac{1}{2} \alpha a_{0}^{2}+2 \beta c^{2} a_{2}+c \gamma a_{1}=0, \\
-v a_{0}+\alpha a_{0} a_{1}-2 a_{1} \beta c^{2}+2 c \gamma a_{2}=0, \\
-v a_{2}+\frac{1}{2} \alpha a_{1}^{2}+a_{0} a_{2}-8 \beta c^{2} a_{2}-c \gamma a_{1}=0, \\
\alpha a_{1} a_{2}+2 \beta c^{2} a_{1}-2 c \gamma a_{2}=0, \\
\frac{1}{2} \alpha a_{2}^{2}+6 \beta c^{2} a_{2}=0 .
\end{array}\right.
$$

Solving the algebraic equations, we get two sets of solutions:

$$
\begin{aligned}
& v=-\frac{6 \gamma^{2}}{25 \beta}, c=-\frac{\gamma}{10 \beta}, a_{0}=-\frac{3 \gamma^{2}}{25 \alpha \beta}, a_{1}=-\frac{6 \gamma^{2}}{25 \alpha \beta}, a_{2}=-\frac{3 \gamma^{2}}{25 \alpha \beta} \\
& v=\frac{6 \gamma^{2}}{25 \beta}, c=-\frac{\gamma}{10 \beta}, a_{0}=\frac{9 \gamma^{2}}{25 \alpha \beta}, a_{1}=-\frac{6 \gamma^{2}}{25 \alpha \beta}, a_{2}=-\frac{3 \gamma^{2}}{25 \alpha \beta} .
\end{aligned}
$$

From (2.2)-(2.4) we obtain two traveling wave solutions of (3.1)

$$
u(x, t)=-\frac{3 \gamma^{2}}{25 \alpha \beta}+\frac{6 \gamma^{2}}{25 \alpha \beta} \tanh \left(\frac{\gamma}{10 \beta}\left(x+\frac{6 \gamma^{2}}{25 \beta} t\right)\right)-\frac{3 \gamma^{2}}{25 \alpha \beta} \tanh ^{2}\left(\frac{\gamma}{10 \beta}\left(x+\frac{6 \gamma^{2}}{25 \beta} t\right)\right)
$$




$$
u(x, t)=\frac{9 \gamma^{2}}{25 \alpha \beta}+\frac{6 \gamma^{2}}{25 \alpha \beta} \tanh \left(\frac{\gamma}{10 \beta}\left(x-\frac{6 \gamma^{2}}{25 \beta} t\right)\right)-\frac{3 \gamma^{2}}{25 \alpha \beta} \tanh ^{2}\left(\frac{\gamma}{10 \beta}\left(x-\frac{6 \gamma^{2}}{25 \beta} t\right)\right)
$$

In a similar way, we can get the following solutions

$$
\begin{aligned}
& u(x, t)=-\frac{3 \gamma^{2}}{25 \alpha \beta}+\frac{6 \gamma^{2}}{25 \alpha \beta} \operatorname{coth}\left(\frac{\gamma}{10 \beta}\left(x+\frac{6 \gamma^{2}}{25 \beta} t\right)\right)-\frac{3 \gamma^{2}}{25 \alpha \beta} \operatorname{coth}^{2}\left(\frac{\gamma}{10 \beta}\left(x+\frac{6 \gamma^{2}}{25 \beta} t\right)\right) \\
& u(x, t)=\frac{9 \gamma^{2}}{25 \alpha \beta}+\frac{6 \gamma^{2}}{25 \alpha \beta} \operatorname{coth}\left(\frac{\gamma}{10 \beta}\left(x-\frac{6 \gamma^{2}}{25 \beta} t\right)\right)-\frac{3 \gamma^{2}}{25 \alpha \beta} \operatorname{coth}^{2}\left(\frac{\gamma}{10 \beta}\left(x+\frac{6 \gamma^{2}}{25 \beta} t\right)\right) \\
&
\end{aligned}
$$
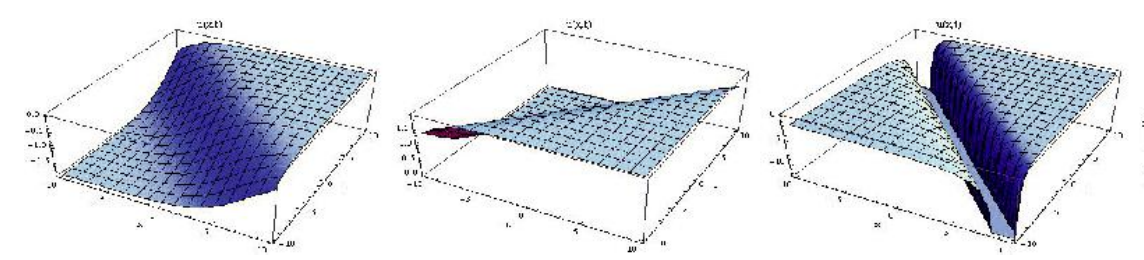

Fig. 1. the travelling wave solutions of (3.3-3.4), when we take $\alpha=\beta=1, \gamma=2$ and

$$
(x, t) \in[-10,10] \times[-10,10] \text {. }
$$

\section{Conclusion}

In this paper, we have applied the tanh-function method to construct a series of traveling wave solutions for some special types of equations: nonlinear beam equations, Fisher-KKP equations and $\mathrm{KdV}$-Burger equations. These traveling waves solutions are expressed in terms of hyperbolic tangent or hyperbolic cotangent functions depending on different pa-rameters. The tanh-function method is direct, concise and effective, which can be applied to many other nonlinear evolution equations.

\section{References}

[1]M.A. Abdou, New exact travelling wave solutions using modified extended tanh-function method, Chaos, Solitons and Fractals 31 (2007), 840-85.

[2]M.J. Ablowitz, P.A. Clarkson, Solitons, Nonlinear Evolution and Inverse Scattering, Cambridge Univ. Press, 1991.

[3]E. Fan, Y. Hon, Generalized tanh method extended to special types of nonlinear equations, Z. Naturforsch 57 (2002) 692-700.

[4]K. Glasner, Nonlinear preconditioning for diffuse interfaces, J. Comput. Phys. 174 (2001), 695-711.

[5]C.H. Gu, Soliton Theory and Its Application, Springer, Berlin, 1995.

[6]D. Huang, D. Li , H. Zhang, Explicit and exact travelling wave solutions for the generalized derivative Schroinger equation, Chaos, Solitons and Fractals 31 (2007), 586-59.

[7]L. Huibin, W. Kelin, Exact solutions for two nonlinear equations: I, J. Phys. A: Math. Gen. 23(1990), 3923-3928.

[8]A.H. Khater, W. Malfliet, D. K. Callebaut, E. S. Kamel, The tanh method, a simple transformation and exact analytical solutions for nonlinear reaction-diffusion equations, Chaos Soliton. Fract 14 (2002), 513-522.

[9]Kamruzzaman Khan, M. Ali Akbar.Traveling Wave Solutions of Some Coupled Nonlinear Evolution Equations.ISRN Mathematical Physics,2013

[10]W. Malfliet, Solitary wave solutions of nonlinear wave equations, Am. J. Phys. 60 (7) (1992), 650-654.

[11]W. Malfliet, The tanh method: I. Exact solutions of nonlinear evolution and wave equations, Physica Scripta 54 (1996), 563-568. 
[12]W. Malfliet, The tanh method: II. Perturbation technique for conservative systems, Physica Scripta 54 (1996), 569-575.

[13]S.A.El-Wakil, A.R.Degheidy, E.M.Abulwafa, M.A.Madkour, M.T.Attia, M.A.Abdou, Exact Travelling Wave Solutions of Generalized Zakharov Equations with Arbitrary Power Nonlinearities, International Journal of Nonlinear Science Vol.7(2009) No.4,pp.455-461

[14]M. Wang, Exact solutions for a compound KdV-Burgers equation, Phys. Lett. A 213 (1998), 279-287.

[15]A.M. Wazwaz, Partial Differential Equations: Methods and Applications, Balkema, TheNetherlands, 2002.

[16]A.M. Wazwaz, The tanh method for traveling wave solutions of nonlinear equations, Applied Mathematics and Computation 154 (2004), 713-72.

[17]PANG Jing,BIAN Chun-quan,CHAO Lu,New Exact Travelling Wave Solutions of Nonlinear Evolution Equations,Journal of Inner Mongolia University.41(2010),13-22

[18]B.Tian, Y. Gao, Truncated Painleve expansion and a wide-ranging type of generalized variable-coefficient Kadomtsev-Petviashvili equations, Phys. Let. A 209 (1995), 297-304.

[19]S.A. El-Wakil,M.A. Madkour, M.A. Abdou.New traveling wave solutions for nonlinear evolution equations,Physics Letters A,365(2007), Issues 5-6, pp. 429-438

[20]Jalil Manafian,Monireh Nasrollahpour.Exact Solutions for the KdV Equation with Forcing Term by the Generalized tanh-coth Method and the -Expansion Method.ISRN Applied Mathematics (2014) 